\title{
A Conserved PLPLRT/SD Motif within the C-terminal Tail of STING Mediates the Recruitment and Activation of TBK1
}

\author{
Pengbiao $\mathrm{Xu}^{1}$, Baoyu Zhao ${ }^{1}$, Pingwei $\mathrm{Li}^{1}{ }^{1,}$ \\ ${ }^{1}$ Department of Biochemistry and Biophysics, Texas A\&M University, College Station, \\ TX 77843, USA \\ * Corresponding Author
}

\section{Email Contact: xpb20081@tamu.edu}

The detection of nucleic acids is a central strategy of innate immunity. Cytosolic DNA is detected by cGAS, which catalyzes the synthesis of a cyclic dinucleotide cGAMP. The adaptor STING binds to cGAMP and mediates the activation of TBK1 and IRF-3. Together, this pathway regulates the induction of type-l interferons. The precise mechanisms governing STING activation by cGAMP and TBK1 activation by STING remain poorly understood. Here we identified a conserved PLPLRT/SD motif within the C-terminal tail of STING that is required for TBK1 recruitment. Crystal structures of TBK1 bound to STING revealed that the PLPLRT/SD motif binds to the dimer interface of TBK1. Cell-based studies confirmed that the direct interaction between TBK1 and STING is essential for IFN- $\beta$ induction upon cGAMP stimulation. To further investigate the mechanism of STING activation, we expressed and purified full-length STING and observed via cryo-EM that STING oligomerizes in the presence of cGAMP.

a

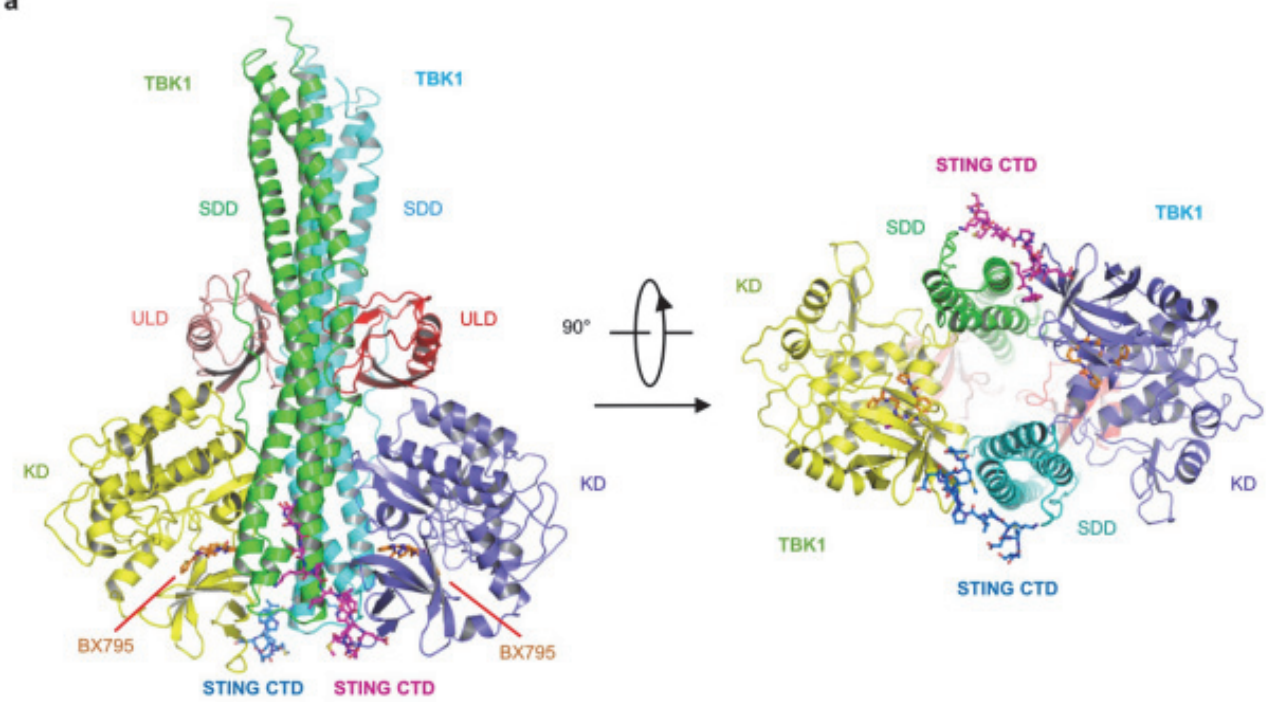

Fig. 1. The crystal structure of TBK1 bound with STING CTD

Reference

[1] Baoyu, Zhao. et al. Nature, in press. 